\title{
Novos Resultados em Partições
}

\author{
Cecília P. Andrade, Kênia C. P. Silva \\ Elen V. P. Silva \\ Instituto de Matemática, Estatística e Computação Científica, IMECC, Unicamp, \\ 13083-859, Campinas, SP \\ E-mail: cissamat@gmail.com, kenia@ime.unicamp.br, elenvps@gmail.com.
}

Resumo: Neste trabalho vamos apresentar resultados obtidos para as Mock Theta Functions $q \omega(q)$ e $f_{0}(q)$, através da representação de uma partição como matriz de duas linhas. Para cada inteiro positivo $n$, geramos todas as matrizes de duas linhas possiveis, somamos suas linhas, resultando em uma matriz de ordem $2 \times 1$ e depois contamos quantas vezes cada soma aparece. Dispomos estes dados em uma tabela e, a partir daí, foi possível conjecturar e demonstrar vários resultados interessantes a respeito de partições. Existem inúmeros resultados que podem ser obtidos e demonstrados combinatorialmente através desta nova estatitisca.

Palavras-chave: Partições, matrizes de duas linhas, Mock Theta Functions

Em [4], foram dadas interpretações como matrizes de duas linhas para as Mock Theta Functions. Baseado nestas representações, introduzimos uma nova estatística, onde, para cada inteiro $n$, geramos todas as matrizes de duas linhas possíveis, somamos suas linhas, de forma que resulte uma matriz de ordem $2 \times 1$ e daí contamos a cardinalidade de tais matrizes. Tabelamos estes valores e então foi possível encontrar alguns resultados.

\section{Mock Theta Function $q \omega(q)$}

De acordo com [4], a função geradora para $q \omega(q)$ é

$$
q \omega(q)=\sum_{n=0}^{\infty} \frac{q^{2 n(n+1)+1}}{\left(q ; q^{2}\right)_{n+1}^{2}}
$$

e a restrição que caracteriza a matriz de duas linhas correspondente é

$$
c_{t}=c_{t+1}+2 d_{t+1}, c_{s}=1 .
$$

Logo, a tabela associada a esta caracterização é a seguinte. 


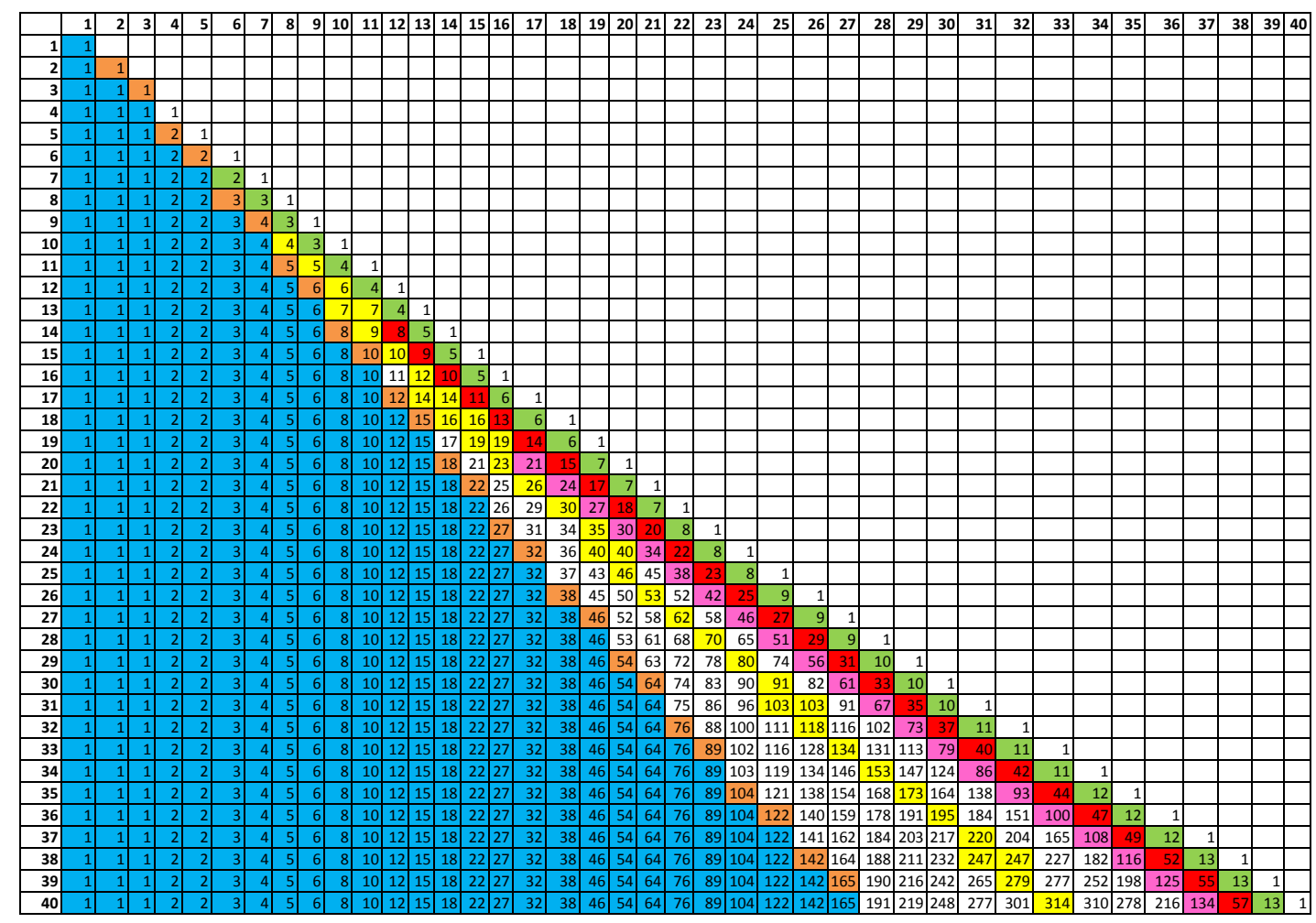

Figura 1: Tabela para $q \omega(q)$

Lema 1.1. Dado $n>0$, o número de matrizes cuja soma é $\left\{\begin{array}{c}n-j \\ j\end{array}\right\}, j \geq 1$, e é da forma

$$
\left(\begin{array}{ccccc}
c_{1} & c_{2} & c_{3} & \ldots & c_{s} \\
d_{1} & d_{2} & d_{3} & \ldots & d_{s}
\end{array}\right)
$$

com as restrições:

$$
\begin{gathered}
c_{s}=1, \\
c_{t}=c_{t+1}+2 d_{t+1}, \\
\sum d_{i}=j, \\
n=\sum c_{t}+\sum d_{t} .
\end{gathered}
$$

é igual ao coeficiente de $x^{n-j}$ na expansão de

$$
\frac{1}{(1-x)\left(1-x^{3}\right)\left(1-x^{5}\right) \cdots\left(1-x^{2 j+1}\right)} .
$$

Ou ainda, fixado $j \geq 1$, seja $P_{2 j+1}(k)$ o número de partições de $k$ em partes ímpares menores que do que ou iguais a $2 j+1$, então

$P_{2 j+1}(n-(j+1))=$ ao número de matrizes cuja soma é $\left\{\begin{array}{c}n-j \\ j\end{array}\right\}, n>j$.

Demonstração. De fato, dada uma matriz da forma (1) podemos associar a partição $c_{1}+c_{2}+$ $\cdots+c_{s-1}$ em partes ímpares menores do que ou iguais a $2 j+1$ de $n-(j+1)$. Reciprocamente, dada uma partição $\lambda_{1}+\cdots+\lambda_{j}$ em partes ímpares menores do que ou iguais a $2 j+1$, em ordem não-crescente, podemos construir a matriz 


$$
\left(\begin{array}{ccccc}
\lambda_{1} & \lambda_{2} & \ldots & \lambda_{j} & 1 \\
d_{1} & d_{2} & \ldots & d_{j} & d_{j+1}
\end{array}\right)
$$

em que $d_{j+1}=\frac{\lambda_{j}-1}{2}, d_{i+1}=\frac{\lambda_{i}-\lambda_{i+1}}{2}$ e $d_{1}=j-\sum_{h=2}^{j+1} d_{h}$.

Proposição 1.1. Dado $m \geq 1$, considere $p_{d}(m)$ o número de partições de $m$ em partes distintas. Então

$$
p_{d}(m)=\#\left\{\begin{array}{c}
m+1 \\
w
\end{array}\right\}, \operatorname{com} w \geq\left\lfloor\frac{m-1}{2}\right\rfloor
$$

Demonstração. Considere $m \geq 1$, para cada partição de $m$ em partes distintas existe uma partição $c_{1}+c_{2}+\cdots+c_{s-1}$ em partes ímpares correspondente. Como demonstrado no Lema (1.1) esta partição pode ser associada à matriz

$$
\left(\begin{array}{ccccc}
c_{1} & c_{2} & \ldots & c_{s-1} & 1 \\
d_{1} & d_{2} & \ldots & d_{s-1} & d_{s}
\end{array}\right)
$$

$\operatorname{com} \sum_{i=1}^{s} d_{i}=w, w \geq\left\lfloor\frac{m-1}{2}\right\rfloor$.

Do mesmo modo, dada uma matriz cuja soma é $\left\{\begin{array}{c}m+1 \\ w\end{array}\right\}$, ela pode ser associada, pelo Lema (1.1), a uma partição de $m$ em partes ímpares menores do que ou iguais a $2 w+1$.

Se $m$ é ímpar então $\left\lfloor\frac{m-1}{2}\right\rfloor=\frac{m-1}{2}$ e portanto $2 w+1 \geq m$. Caso contrário, teremos $\left\lfloor\frac{m-1}{2}\right\rfloor=\frac{m-2}{2}$ e então $2 w+1 \geq m-1$, e como $m$ é par, segue em ambos os casos, que temos partições em partes ímpares irrestritas. Mas como para cada partição de $m$ em partes ímpares temos uma partição em partes distintas segue o resultado.

\section{Mock Theta Function $f_{0}(q)$}

A função geradora para $f_{0}(q)$, segundo [4], é

$$
f_{0}(q)=\sum_{n=0}^{\infty} \frac{q^{n^{2}}}{(-q ; q)_{n}}
$$

e a restrição que caracteriza a matriz de duas linhas correspondente é

$$
c_{t}=2+c_{t+1}+d_{t+1}, c_{s}=1 .
$$

Esta função tem um peso associado, dado por $(-1)^{\sum_{t=1}^{s} d_{t}}$. Considere a tabela da Mock Theta Function $f_{0}(q)$. 


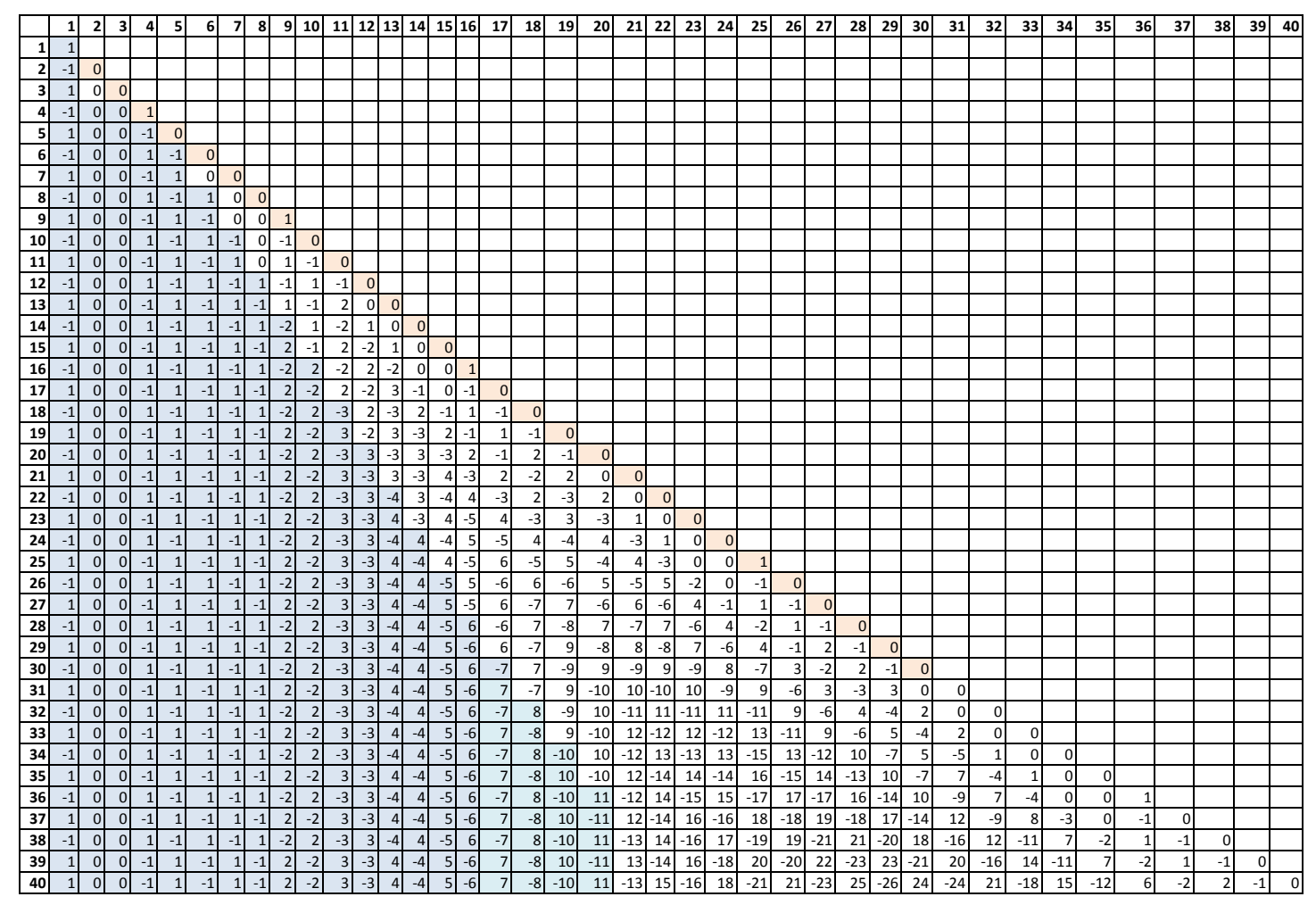

Figura 2: Tabela para $f_{0}(q)$

Quando calculamos todas as matrizes possíveis e fazemos seu gráfico de Ferrers, observamos que cada partição não possui pontos à direita do quadrado de Durfee, e além disso, para cada inteiro $2 n-4$, existem exatamente $n-4$ partes abaixo do quadrado de Durfee. Isto nos sugere o seguinte resultado.

Teorema 2.1. O número de partições de um inteiro positivo $2 n-4$ que não possuem pontos à direita do quadrado de Durfee e possuem exatamente $n-4$ partes abaixo do quadrado de Durfee é igual ao número de matrizes de duas linhas na forma

$$
\left(\begin{array}{ccccc}
c_{1} & c_{2} & c_{3} & \cdots & c_{k} \\
d_{1} & d_{2} & d_{3} & \cdots & d_{k}
\end{array}\right)
$$

em que

$$
\begin{aligned}
c_{k} & =1 \\
c_{t} & =2+c_{t+1}+d_{t+1}, t<k \\
\sum_{i=1}^{k} c_{i}+d_{i} & =2 n-4 ; \\
\sum_{i=1}^{k} c_{i} & =n \\
\sum_{i=1}^{k} d_{i} & =n-4 .
\end{aligned}
$$

Demonstração. A prova deste teorema segue de modo análogo ao Corolário $C$ em [2]. É suficiente construir uma bijeção do conjunto de partições de $2 n-4$ com as restrições descritas no Teorema (2.1) e o conjunto de matrizes da forma (3), em que associa a cada $k \geq 1$ fixado, uma partição de $2 n-4$ com nenhum ponto à direita do quadrado de Durfee de lado $k$ a uma matriz com as restrições (3), contendo exatamente $k$ colunas de forma que $d_{1}$ é o número de partes iguais a 1 
abaixo do quadrado de Durfee, $d_{2}$ é o número de partes iguais a 2 abaixo do quadrado de Durfee e assim por diante.

Por exemplo, uma partição $\lambda=\lambda_{1}+\lambda_{2}+\ldots+\lambda_{r}$ (suponha $\lambda_{t} \geq \lambda_{t+1}$ ) com quadrado de Durfee de lado 3 é completamente caracterizada, uma vez que for declarado quantas vezes cada um dos números 1, 2 e 3 aparece como parte de $\lambda$. Seja

$$
\left(\begin{array}{ccc}
c_{1} & c_{2} & c_{3} \\
d_{1} & d_{2} & d_{3}
\end{array}\right)
$$

uma matriz. Por (3) é possível escrever

$$
\begin{aligned}
& c_{3}=1 \\
& c_{2}=3+d_{3} \\
& c_{1}=5+d_{2}+d_{3},
\end{aligned}
$$

Portanto, a matriz (4) pode ser escrita como

$$
\left(\begin{array}{ccc}
5+d_{2}+d_{3} & 3+d_{3} & 1 \\
d_{1} & d_{2} & d_{3}
\end{array}\right)
$$

ou, ainda, como a soma

$$
\left(\begin{array}{lll}
5 & 3 & 1 \\
0 & 0 & 0
\end{array}\right)+\left(\begin{array}{ccc}
0 & 0 & 0 \\
d_{1} & 0 & 0
\end{array}\right)+\left(\begin{array}{ccc}
d_{2} & 0 & 0 \\
0 & d_{2} & 0
\end{array}\right)+\left(\begin{array}{ccc}
d_{3} & d_{3} & 0 \\
0 & 0 & d_{3}
\end{array}\right)
$$

Da discussão anterior, segue que a partição $\lambda$ pode ser caracterizada pelos números $d_{1} \rightarrow$ o número de vezes que 1 é uma parte de $\lambda$ $d_{2} \rightarrow$ o número de vezes que 2 é uma parte de $\lambda$ $d_{3} \rightarrow$ o número de vezes que 3 é uma parte de $\lambda$.

Exemplo 2.1. Dada a matriz $\left(\begin{array}{ccc}13 & 3 & 1 \\ 5 & 8 & 0\end{array}\right)$, podemos associá-la ao gráfico de Ferrers com quadrado de Durfee de tamanho 3 e tendo 8 partes de tamanho 2 e 5 partes de tamanho 1.

$$
\begin{aligned}
& \left(\begin{array}{ccc}
13 & 3 & 1 \\
5 & 8 & 0
\end{array}\right)=\left(\begin{array}{lll}
5 & 3 & 1 \\
0 & 0 & 0
\end{array}\right)+8 \cdot\left(\begin{array}{ll}
1 & 0 \\
0 & 1
\end{array}\right)+5 \cdot\left(\begin{array}{l}
0 \\
1
\end{array}\right)= \\
& \text { - } \bullet \\
& \text { - } . \\
& \text { - } \bullet \\
& \text { - } \\
& \text { - } \\
& \text { - } \\
& \text { - } \\
& \text { - } \\
& \text { - } \\
& \text { - } \\
& \text { - } ・ \\
& \text { - } \\
& \text { - } \\
& \text { - }
\end{aligned}
$$

Observamos que até um certo ponto os valores vão se repetindo de uma linha para outra na tabela e a cada duas linhas um valor é acrescentado nesta sequência (sequência em azul). De acordo com Sloane [6], esta é a sequência das partições balanceadas de $n$. 
Definição 2.1. Uma partição balanceada de um inteiro positivo n é aquela em que a última parte é igual ao número de partes.

Lema 2.1. Seja $n$ inteiro positivo e $\lambda$ uma partição de $n$ balanceada com quadrado de Durfee de tamanho $k \geq 1$. Então a partição conjugada de $\lambda$ tem um número menor do que ou igual a $n-4$ partes abaixo do quadrado de Durfee.

Demonstração. Considere $n$ um inteiro positivo, e $\lambda$ uma partição balanceada de $n$,com quadrado de Durfee de tamanho $k \geq 1$.

Se $k=1$ nada temos a fazer, pois somente para $n=1$ é possível ter uma partição balanceada.

Considere $k \geq 2$. Como $n-k^{2} \leq n-2^{2}=n-4$, além do quadrado de Durfee de tamanho $k^{2}$, resta um número menor do que ou igual a $n-4$ de pontos na partição conjugada de $\lambda$.

Baseado na observação e no lema anterior, temos o seguinte resultado.

Teorema 2.2. O número de partições balanceadas de um inteiro positivo $n \geq 7$ é igual ao número de matrizes de duas linhas na forma

$$
\left(\begin{array}{ccccc}
c_{1} & c_{2} & c_{3} & \cdots & c_{k} \\
d_{1} & d_{2} & d_{3} & \cdots & d_{k}
\end{array}\right)
$$

em que

$$
c_{k}=1,
$$$$
c_{t}=2+c_{t+1}+d_{t+1}, t<k
$$

$$
\begin{gathered}
\sum_{i=1}^{k} c_{i}+d_{i}=2 n-4 ; \\
\sum_{i=1}^{k} c_{i}=n ; \\
\sum_{i=1}^{k} d_{i}=n-4 .
\end{gathered}
$$

Demonstração. Seja $n \geq 7$ inteiro positivo e $\lambda$ uma partição balanceada de $n$ com quadrado de Durfee de tamanho $k \geq 1$. Então conjugamos a partição $\lambda$, deslocamos as partes abaixo do quadrado de Durfee uma posição para a direita e completamos com uma coluna de tamanho $n-4$. Observe que é possível completar, pois o Lema (2.1) garante que existe um número menor do que $n-4$ partes abaixo do quadrado de Durfee e nenhuma dessas partes é igual ao tamanho do quadrado, pela definição de partição balanceada. Desta forma construímos uma partição de $2 n-4$, sem pontos à direita do quadrado de Durfee, que pelo Teorema (2.1) está associada a uma matriz do tipo (3).

Reciprocamente, dada uma matriz da forma (3), podemos associar a partição de $2 n-4$ com exatamente $n-4$ partes abaixo e sem nenhum ponto à direita do quadrado de Durfee, a uma partição de $n$ também com quadrado de Durfee de tamanho $k$. Primeiro retiram-se $n-4$ pontos, um ponto de cada parte abaixo do quadrado de Durfee na representação de Ferrers da partição de $2 n-4$ e depois conjuga-se a partição. Portanto o número de partes é exatamente o tamanho do quadrado de Durfee e é igual à menor parte, o que resulta numa partição balanceada de $n$.

Existem inúmeros resultados que podem ser obtidos e demonstrados combinatoriamente através desta nova estatítisca. 


\section{Referências}

[1] G.E. Andrews; Integer Partitions, Cambridge University Press, 2004..

[2] E.H.M. Brietzke, J.P.O. Santos, R. Silva, Bijective proofs using two-line matrix representations for partitions, The Ramanujan Jornal, V23, p. 265-295, 2010.

[3] E.H.M. Brietzke, J.P.O. Santos, R. Silva, A new approach and generalizations to some results about mock theta functions, Discrete Mathematics, v. 311, p. 595-615, 2011.

[4] E.H.M. Brietzke, J.P.O. Santos, R. Silva, Combinatorial interpretations as two-line array for the mock theta functions, Bulletin of the Brazilian Mathematical Society (Impresso), v. 44, p. 233-253, 2013.

[5] J.P.O. Santos, P. Mondek, A.C. Ribeirto, New Two-Lines Arrays Representing Partitions, Annals of Combinatorics, v. 15, p. 341-354, 2011.

[6] https://oeis.org/ 